\title{
WEAK CONTAINMENT AND INDUCED REPRESENTATIONS OF GROUPS. II(')
}

BY

J. M. G. FELL

Introduction. In [6] we have outlined a program for determining the structure of an arbitrary $C^{*}$-algebra $A$ in terms of its primitive images. The first step in this program is always to determine the topological space $\hat{A}$ of all equivalence classes of irreducible representations of $A$, with the hull-kernel topology. If $A$ is the group $C^{*}$-algebra of a locally compact group, $A$ becomes the topological space $\hat{G}$ of equivalence classes of irreducible unitary representations of $G$. How is $\hat{G}$ to be analyzed? By Mackey's general theory [17], the elements of $\hat{G}$ are obtained (under certain broad conditions) by inducing from representations of subgroups. It would be desirable to supplement Mackey's theory so as to obtain not merely the elements of $\hat{G}$ but also their topology, in terms of the corresponding entities on subgroups. As a first step in this program, we proved in [5] the continuity of the process of inducing a representation of a subgroup $K$ up to a representation of $G$ [5, Theorem 4.1]. However, this kind of theorem, in which the subgroup $K$ is fixed, is inadequate; we need to consider the case in which not only the representation of $K$ but even $K$ itself can vary continuously (see the last page of [5]). The main purpose of the present paper is to formulate and prove such a generalization of Theorem 4.1 of [5]. We shall introduce a topology into the set $\mathscr{S}(G)$ of all pairs $\langle K, T\rangle$, where $K$ is a (variable) closed subgroup of $G$, and $T$ is a unitary representation of $K$; and then prove (Theorem 4.2) that the result ${ }_{L} U^{T}$ of inducing the representation $T$ of $K$ up to a representation of a subgroup $L$ containing $K$, is continuous in all three variables $T, K$, and $L$. A similar theorem holds for the operation of restriction (Theorem 3.2): The result $T \mid L$ of restricting $T$ to a subgroup $L$ contained in $K$ is continuous in $T, K$, and $L\left(^{2}\right)$.

Recently Glimm, in [11], has substantially advanced the program of determining the topology of $\hat{G}$. The continuity of ${ }_{L} U^{T}$ as a function of $\langle K, T\rangle$, the "supergroup" $L$ being fixed, follows easily from the results of [11]. This does not seem to be the case, however, if $L$ also is allowed to vary. At any rate, we do not presuppose the results of [11] (except for the existence of "smooth choices" of Haar measure, see $\$ 1$ ).

Received by the editors January 20, 1963.

(1) This work was partially supported by NSF Grant No. G-17892.

(2) This generalizes $[4$, p. 371 , Corollary]. 
The contents of the six sections of this paper are as follows. $G$ is a fixed locally compact group.

In $\S 1$ we list certain known, or almost known, facts concerning the inner hull-kernel topology of representations of a $C^{*}$-algebra or locally compact group. Also we recall from [7] the definition of the compact-open topology of the space $\mathscr{K}(G)$ of all closed subgroups of $G$, and from [11] the existence of a "smooth choice", of Haar measures on $\mathscr{K}(G)$.

In $\$ 2$ the "subgroup algebra" of $G$ is defined (by a construction found in [11]). This is a Banach *-algebra with a natural "fibering"; the base space of the fibering is $\mathscr{K}(G)$, and the fiber at $K$ is the group algebra of $K$. Using this, we introduce a topology into the set $\mathscr{S}(G)$ defined above, and examine its elementary properties. This leads to another natural topology for $\mathscr{K}(G)$, called the representational topology. We do not know whether this can ever differ from the compactopen topology for $\mathscr{K}(G)$.

$\$ 3$ gives an equivalent description of the topology of $\mathscr{S}(G)$ in terms of functions of positive type on subgroups; this generalizes Theorem 1.3 of [4]. As a corollary we obtain the continuity of the restriction operation with varying subgroups (Theorem 3.2). In $\S 4$ we prove our main theorem, on the continuity of the operation of inducing a representation $T$ of a smaller subgroup $K$ up to a bigger subgroup $L$; here $T, K$, and $L$ all vary simultaneously.

Some work has been done (see $[15 ; 18]$ ) on generalizing the classical Frobenius Reciprocity Theorem to noncompact groups. Given $G$ and a closed subgroup $K$, one can contemplate the following "topological Frobenius property": For each $T$ in $\hat{K}$ and $S$ in $\hat{G},{ }_{G} U^{T}$ weakly contains $S$ if and only if $S \mid K$ weakly contains $T$. This is far from holding in general. In $\S 5$ we prove weak forms of the above property for certain special cases. In so doing, we use $\$ 4$ to derive a topological version (Theorem 5.2) of Mackey's Restriction Theorem [14, Theorem 12.1].

Finally, $\$ 6$ gives a counter-example to a very weak Frobenius-like property Suppose $K$ is a closed subgroup of $G$ and $f$ is a function of positive type on $K$. It is well known that it may not be possible to extend $f$ to a function of positive type on $G$. This counter-example shows that it may not even be possible to approximate $f$, uniformly on compact sets, by restrictions to $K$ of functions of positive type on $G$.

1: Notation and preliminary information. The void set will be called $\Lambda$. We write $\langle x, y\rangle$ for ordered pairs, and $(x, y)$ for inner products. The domain of a function $f$ is $D(f)$; the restriction of $f$ to a subset $E$ of $D(f)$ is $f \mid E$. If $f$ is complexvalued, $\|f\|_{\infty}=\sup \{|f(x)| \mid x \in D(f)\}$. If $T$ is a unitary representation of a closed subgroup $K$ of a locally compact group $G,{ }_{G} U^{T}$ (or $U^{T}$ if no confusion can arise) is the unitary representation of $G$ induced from $T$. For the theory of induced representations, see [14] and [2] (the latter for the nonseparable situation). 
If $X$ is a locally compact Hausdorff space, $C(X)$ will be the space of all continuous complex functions on $X$, and $C_{0}(X)$ the subspace of $C(X)$ consisting of the functions with compact support.

If $A$ is a Banach *-algebra, a ${ }^{*}$-representation of $A$ is a nowhere trivial( $\left.{ }^{3}\right)$ *-homomorphism $T$ of $A$ into the *-algebra of all bounded linear operators on some Hilbert space $H(T)$, called the space of $T$. If $A$ has enough *-representations to distinguish points, it is reduced; in that case we can define a new norm \|\|$_{c}$ for $A$ (the minimal regular norm, see [19]):

$$
\|a\|_{c}=\sup \left\|T_{a}\right\|
$$

(where the supremum is taken over all *-representations $T$ of $A$ ). The completion of $A$ with respect to \|\|$_{c}$ is a $C^{*}$-algebra, the $C^{*}$-completion of $A$. The *-representations of $A$ and of its $C^{*}$-completion are essentially identical. If $G$ is a locally compact group with left Haar measure $\mu$, the $C^{*}$-completion of $L_{1}(G, \mu)$ is called the group $C^{*}$-algebra of $G$, and is denoted by $C^{*}(G)$. We shall usually use the same letter to denote a unitary representation of $G$ and its "integrated form" (the latter being a *-representation of $L_{1}(G, \mu)$ or $C^{*}(G)$ ). The set of all unitary equivalence classes of *-representations of a Banach *-algebra $A$ is called $\mathscr{T}(A)$; the set of all irreducible elements of $\mathscr{T}(A)$ is called $\hat{A}$ (the dual space of $A$ ). Similarly we define $\mathscr{T}(G)$ and $\hat{G}$ for a locally compact group $G$.

Let $A$ be a fixed $C^{*}$-algebra. If $T \in \mathscr{T}(A)$ and $\mathscr{S} \subset \mathscr{T}(A), T$ is weakly contained $\left.{ }^{4}\right)$ in $\mathscr{S}$ if

$$
\operatorname{Kernel}(T) \supset \bigcap_{S \in \mathscr{S}} \operatorname{Kernel}(S),
$$

or equivalently( $\left.{ }^{5}\right)$, if every positive functional associated with $T$ can be weakly* -approximated by sums of positive functionals associated with $\mathscr{S}$. Two subsets $\mathscr{T}_{1}$ and $\mathscr{S}_{2}$ of $\mathscr{T}(A)$ are weakly equivalent if every $S$ in $\mathscr{S}_{1}$ is weakly contained in $\mathscr{S}_{2}$, and conversely. Restricted to $\hat{A}$, the relation of weak containment defines the closure operation for the hull-kernel topology of $\hat{A}$. Each $T$ in $\mathscr{T}(A)$ is weakly equivalent to a unique closed subset $\operatorname{Sp}(T)$ of $\hat{A}$, called the spectrum of $T$. If $\mathscr{F}$ is a finite family of ncnvoid open subsets of $\hat{A}$, let $U(\mathscr{F})=\{T \in \mathscr{T}(A) \mid \operatorname{Sp}(T) \cap B \neq \Lambda$ for each $B$ in $\mathscr{F}\}$. The inner hull-kernel topology of $\mathscr{T}(A)$ is the topology in which the set of all $U(\mathscr{F})(\mathscr{F}$ being as above) forms a basis of open sets $\left(^{6}\right)$. Relativized to $\hat{A}$, this is again the hull-kernel topology.

In this paper we always assume that $\mathscr{T}(A)$ and $\hat{A}$ are equipped with the inner hull-kernel and hull-kernel topologies, respectively.

(3) $T$ is nowhere trivial if the linear span of $\left\{T_{a} v \mid a \in A, v \in H(T)\right\}$ is dense in $H(T)$. The zero representation, with zero-dimensional $H(T)$, is admitted as nowhere trivial, but not as irreducible. The zero representation is also admitted as a unitary, but not as an irreducible, representation of a locally compact group.

(4) For this and the concepts that follow, see [4] and [5].

(5) [4, Theorem 1.2].

(6) This topology is of course wildly non-Hausdorff. 
In view of Theorems 1.1 and 2.3 of [5] and the remark following Theorem 2.1 of [5], we have:

Proposition 1.1. If $T \in \mathscr{T}(A)$ and $\mathscr{S} \subset \mathscr{T}(A)$, the following are equivalent: (a) $T$ is weakly contained in $\mathscr{S}$; (b) the closure of $\bigcup_{S \in \mathscr{S}} \mathrm{Sp}(S)$ contains $\operatorname{Sp}(T)$; (c) $T$ is in the closure of the set $\mathscr{S}_{f}$ of all finite direct sums of elements of $\mathscr{S}$. If $T$ is irreducible, these conditions hold if and only if $T$ belongs to the closure of $\mathscr{S}$.

The following easily verifiable facts will also be useful:

Proposition 1.2. A net $\left\{T^{i}\right\}$ converges to $T$ in $\mathscr{T}(A)$ if and only if every subnet of $\left\{T^{i}\right\}$ weakly contains $T$.

Proposition 1.3. If $T^{i} \rightarrow T$ in $\mathscr{T}(A)$ and $T$ weakly contains $S$, then $T^{i} \rightarrow S$ in $\mathscr{T}(A)$.

Proposition 1.4( $\left.{ }^{7}\right) . \quad T^{i} \rightarrow T$ in $\mathscr{T}(A)$ if and only if $\left\|T_{a}\right\| \leqq \liminf _{i}\left\|T_{a}^{i}\right\|$ for each $a$ in $A$.

Proposition 1.5. If $T \in \mathscr{T}(A)$ and $T$ is weakly contained in $\{S \in \mathscr{T}(A) \mid$ $\operatorname{Kernel}(S) \supset I\}$, where $I$ is a closed two-sided ideal of $A$, then $\operatorname{Kernel}(T) \supset I$.

Proposition 1.6. Suppose $M$ is a group of *automorphisms of $A$ with the topology of pointwise convergence on $A$. Then the map $\langle T, V\rangle \rightarrow T \circ V$ is continuous on $\mathscr{T}(A) \times M$ to $\mathscr{T}(A)$.

If $A$ is a locally compact group or a reduced Banach *-algebra, we define weak containment, the inner hull-kernel topology, and so forth, for $\mathscr{T}(A)$ by passing to the group $C^{*}$-algebra (or $C^{*}$-completion), and identifying $\mathscr{T}(A)$ with the family of all representations of the latter.

Let $X$ be an arbitrary locally compact ${ }^{8}$ ) (not necessarily Hausdorff) space. As in [7] we equip the family $\mathscr{X}(X)$ of all closed subsets of $X$ with the compactopen topology $\left({ }^{9}\right)$ as follows: For each compact subset $C$ of $X$ and each finite family $\mathscr{F}$ of nonvoid open subsets of $X$, let $U(C ; \mathscr{F})=\{Y \in \mathscr{X}(X) \mid Y \cap C=\Lambda$, $Y \cap B \neq \Lambda$ for each $B$ in $\mathscr{F}\}$. A subset $\mathscr{Y}$ of $\mathscr{X}(X)$ is open in the compact-open topology if and only if it is a union of certain of the $U(C ; \mathscr{F})(C, \mathscr{F}$ being as above). With this topology $\mathscr{X}(X)$ is a compact Hausdorff space $\left({ }^{10}\right)$.

Let $A$ be a $C^{*}$-algebra. Since $\hat{A}$ is locally compact( ${ }^{(1)}, \mathscr{X}(\hat{A})$ is compact in the compact-open topology. If we identify each $T$ with $\operatorname{Sp}(T)$, the inner hull-kernel topology of $\mathscr{T}(A)$ is contained in the compact-open topology of $\mathscr{X}(\hat{A})$. Hence we have:

(7) This follows from Proposition 1.2 above and Lemma 2.1 of [4].

(8) "Locally compact" means that every point has a basis of compact neighborhoods.

(9) In [7] we called this the $H$-topology.

(10) $[7$, Theorem 1$]$.

(11) $[6$, Theorem 2.1]. 
Proposition 1.7. If $A$ is a locally compact group or a reduced Banach *algebra, $\mathscr{T}(A)$ is compact (in the inner hull-kernel topology).

In this paper $G$ will always be a fixed locally compact group with unit $e\left({ }^{12}\right)$. Let $\mathscr{K}(G)$ be the family of all closed subgroups of $G$. As a subset of $\mathscr{X}(G), \mathscr{K}(G)$ is closed in the compact-open topology. We shall assume, unless the contrary is explicitly stated, that $\mathscr{K}(G)$ carries the relativized compact-open topology. Thus $\mathscr{K}(G)$ is a compact Hausdorff space.

A choice of Haar measures in $\mathscr{K}(G)$ is a mapping $K \rightarrow \mu_{K}$ assigning to each $K$ in $\mathscr{K}(G)$ a left Haar measure on $K$. Such a choice is smooth if, for each $f$ in $C_{0}(G)$, the function $K \rightarrow \int_{K} f(x) d \mu_{K} x$ is continuous on $\mathscr{K}(G)$. It is known from [11] that smooth choices exist. Indeed, if $g$ is a fixed non-negative-valued function in $C_{0}(G)$ with $g(e)>0$, and $\mu_{K}$ is chosen for each $K$ so that $\int_{K} g(x) d \mu_{K} x=1$, then $\left\{\mu_{K}\right\}$ is a smooth choice. This leads to an equivalent characterization of the compact-open topology of $\mathscr{K}(G)$ (which we shall not need) in terms of the weak convergence of Haar measures on the subgroups.

It is easily verified that any two smooth choices $\left\{\mu_{K}\right\}$ and $\left\{\mu_{K}^{\prime}\right\}$ are connected by a positive multiplicative factor $\alpha$ (that is, $\mu_{K}^{\prime}=\alpha(K) \mu_{K}$ ) which is continuous on $\mathscr{K}(G)$.

2. A topology for representations of subgroups. Inspired by Glimm [11], we now construct the "subgroup algebra" $A_{s}(G)$ of $G$. Let $Y$ be the set of all pairs $\langle K, x\rangle$, where $K \in \mathscr{K}(G)$ and $x \in K$. It is easily seen that $Y$ is a closed subset of $\mathscr{K}(G) \times G$, hence itself locally compact in the relativized topology. Let us fix once for all a smooth choice $\left\{\mu_{K}\right\}$ of Haar measures on $\mathscr{K}(G)$. Lemma 1.1 of [11] gives:

LEMMA 2.1. If $f \in C_{0}(Y)$, the function

is continuous on $\mathscr{K}(G)$.

$$
K \rightarrow \int_{K} f(K, x) d \mu_{K} x
$$

Let $\Delta_{K}$ be the modular function for the closed subgroup $K$. Arguing as in $[11]\left({ }^{13}\right)$, we show that $\langle K, x\rangle \rightarrow \Delta_{K}(x)$ is continuous on $Y$. We now make $C_{0}(Y)$ into a normed *-algebra as follows: If $f, g \in C_{0}(Y)$,

$$
\begin{aligned}
\left(f_{*} g\right)(K, x) & =\int_{K} f(K, y) g\left(K, y^{-1} x\right) d \mu_{K} y, \\
f^{*}(K, x) & =\overline{f\left(K, x^{-1}\right)} \Delta_{K}\left(x^{-1}\right), \\
\|f\| & =\sup _{K \in \mathscr{K}(G)} \int_{K}|f(K, x)| d \mu_{K} x .
\end{aligned}
$$

(12) $G$ need not satisfy the second axiom of countability until $\S 5$.

(13) See the paragraph following Lemma 1.1 in [11]. 
Each element of $C_{0}(Y)$ can be thought of as a function on $\mathscr{K}(G)$ whose value at $K$ is in the group algebra of $K$. The operations in $C_{0}(Y)$, looked at in this light, are pointwise; clearly $C_{0}(Y)$ is a normed *-algebra (satisfying $\left.\left\|f^{*}\right\|=\|f\|\right)$. The completion $A_{s}(G)$ of $C_{0}(Y)$ with respect to this norm is a Banach *-algebra called the subgroup algebra of $G$.

For each $K$ in $\mathscr{K}(G)$ the mapping $f \rightarrow f_{K}$, initially defined on $C_{0}(Y)$ by $f_{K}(x)=f(K, x)$, extends to a continuous *-homomorphism, which we shall call $\Phi_{K}$, of $A_{s}(G)$ onto a dense subalgebra of $L_{1}\left(K, \mu_{K}\right)$. The following lemma is easy to verify:

Lemma 2.2. For each $f$ in $A_{s}(G), K \rightarrow\left\|\Phi_{K}(f)\right\|$ is continuous on $\mathscr{K}(G)$ and $\|f\|=\sup _{K}\left\|\Phi_{K}(f)\right\|$.

Each unitary representation $T$ of a closed subgroup $K$ can be lifted to a *-representation $W^{K, T}$ of $A_{s}(G)$ thus $\left({ }^{14}\right): W^{K, T}=T \circ \Phi_{K}$. It follows from this and Lemma 2.2 that $A_{s}(G)$ is a reduced Banach *-algebra. Its $C^{*}$-completion, denoted by $C_{s}^{*}(G)$, is called the subgroup $C^{*}$-algebra of $G$; the norm of $C_{s}^{*}(G)$ will be denoted by \|\|$_{c}$. Corresponding representations of $A_{s}(G)$ and $C_{s}^{*}(G)$ will be designated by the same letter. A representation of the form $W^{K, T}$ will be said to be lifted from $K$.

Let $\mathscr{S}(G)$ be the set of all subgroup representations, that is, pairs $\langle K, T\rangle$ where $K \in \mathscr{K}(G)$ and $T \in \mathscr{T}(K)$; we agree to identify all pairs $\langle K, T\rangle$ for which the representation $T$ is identically zero $\left({ }^{15}\right)$ (the resulting element being called the zero element $\theta$ of $\mathscr{S}(G)$ ). By the inner hull-kernel topology of $\mathscr{S}(G)$ we mean that topology which makes the one-to-one mapping $\langle K, T\rangle \rightarrow W^{K, T}$ a homeomorphism with respect to the inner hull-kernel topology of $\mathscr{T}\left(A_{s}(G)\right)$. This is the only topology of $\mathscr{S}(G)$ which we shall use. Here are some of its more evident properties:

Lemma 2.3. The topology of $\mathscr{S}(G)$ is independent of the particular smooth choice of Haar measures $\left\{\mu_{K}\right\}$.

Proof. Let $\left\{\mu_{K}^{\prime}\right\}$ be another smooth choice, connected with $\left\{\mu_{K}\right\}$ by a factor $\alpha$ which is continuous on $\mathscr{K}(G)$ (see end of $\S 1$ ). Let $A_{s}^{\prime}(G)$ and $\left(W^{\prime}\right)^{K, T}$ be the corresponding constructs. The mapping $F$ of $C_{0}(Y)$ onto itself, defined by

$$
F(f)(K, x)=\frac{1}{\alpha(K)} f(K, x)
$$

extends to an isometric isomorphism of $A_{s}(G)$ onto $A_{s}^{\prime}(G)$ and carries $W^{K, T}$ into $\left(W^{\prime}\right)^{K, T}$. This proves the lemma.

(14) Recall that the integrated form of $T$ is also called $T$.

(15) See footnote 3. 
Lemma 2.4. $\left\{W^{K, T} \mid\langle K, T\rangle \in \mathscr{S}(G)\right\}$ is closed in $\mathscr{T}\left(A_{s}(G)\right)$. Thus $\mathscr{P}(G)$ is compact.

Proof. Suppose that $\left\{W^{K^{i}, T^{i}}\right\}$ converges to $S$ in $\mathscr{T}\left(A_{s}(G)\right)$. To prove the first part, we must show that $S$ is lifted from some subgroup $K$. By the compactness of $\mathscr{K}(G)$ we may pass to a subnet and assume $K^{i} \rightarrow K$ in $\mathscr{K}(G)$. Then, for any $f$ in $A_{s}(G)$ with $f_{K}=0\left({ }^{16}\right)$, we have by Proposition 1.4 and Lemma 2.2

$$
\begin{aligned}
\left\|S_{f}\right\| & \leqq \underset{i}{\liminf }\left\|W_{f}^{K^{i}, T^{i}}\right\| \leqq \liminf _{i}\left\|T^{i}\left(f_{K^{i}}\right)\right\| \\
& \leqq \liminf _{i}\left\|f_{K^{i}}\right\|=0 .
\end{aligned}
$$

So $S_{f}=0$. Thus $\operatorname{Kernel}\left(\Phi_{K}\right) \subset \operatorname{Kernel}(S)$, and $S$ is lifted from $K$.

From this and Proposition 1.7 follows the compactness of $\mathscr{S}(G)$.

LEMMA 2.5. The mapping $\pi:\langle K, T\rangle \rightarrow K$ is continuous on $\mathscr{S}(G)-\{\theta\}$ to $\mathscr{K}(G)$.

Proof. Let $\mathscr{L}$ be a closed subset of $\mathscr{K}(G)$, and let $I$ be the closure in $C_{s}^{*}(G)$ of $\left\{f \in C_{0}(Y) \mid f(K, x)=0\right.$ whenever $\left.\langle K, x\rangle \in Y, K \in \mathscr{L}\right\}$. Then

$$
\mathscr{I}=\left\{S \in \mathscr{T}\left(C_{s}^{*}(G)\right) \mid \operatorname{Kernel}(S) \supset I\right\}
$$

is closed in $\mathscr{T}\left(C_{s}^{*}(G)\right)$ by Proposition 1.5. Hence

$$
\left\{\langle K, T\rangle \mid W^{K, T} \in \mathscr{I}\right\}=\pi^{-1}(\mathscr{L}) \cup\{\theta\}
$$

is closed in $\mathscr{S}(G)$.

It seems to be unknown whether $\pi$ is open in general. Some equivalent conditions for openness of $\pi$ will be found in Theorem 2.1 .

LemMA 2.6. For each $K$ in $\mathscr{K}(G)$, the mapping $T \rightarrow\langle K, T\rangle$ is a homeomorphism of $\mathscr{T}(K)$ into $\mathscr{S}(G)$.

Proof. $\Phi_{K}$ may be regarded as a *-homomorphism of $C_{s}^{*}(G)$ onto $C^{*}(K)$; and the map $T \rightarrow W^{K, T}$ amounts to lifting a representation of $C^{*}(K)$ to one of $C_{s}^{*}(G)$ via $\Phi_{K}$. This is clearly a homeomorphism.

LEMMA 2.7. Let $M$ be a topological group acting continuously on $G\left({ }^{17}\right)$. Let $\beta$ be the corresponding action of $M$ on $\mathscr{S}(G): \beta_{m}(\langle K, T\rangle)=\left\langle m \cdot K, T^{\prime}\right\rangle$ $(m \in M)$, where $T_{x}^{\prime}=T_{m^{-1}{ }_{x}}(x \in m \cdot K)$. Then $\beta$ is continuous on $M \times \mathscr{S}(G)$ to $\mathscr{P}(G)$.

Proof. For each $m$ in $M$ and $K$ in $\mathscr{K}(G)$, let $\alpha(m, K)$ be the positive number such that, for each Borel set $E, \mu_{K}\left(m^{-1} \cdot E\right)=\alpha(m, K) \mu_{m \cdot K}(E)$. The relation

(16) We sometimes write $f_{K}$ for $\Phi_{K}(f)$, for arbitrary $f$ in $A_{s}(G)$.

(17) That is, $\langle m, x\rangle \rightarrow m \cdot x$ is continuous on $M \times G$ to $G, m \cdot\left(x_{1} x_{2}\right)=\left(m \cdot x_{1}\right)\left(m \cdot x_{2}\right)$, $\left(m_{1} m_{2}\right) \cdot x=m_{1} \cdot\left(m_{2} \cdot x\right)$, and $e \cdot x=x$ for all $x$. 


$$
\alpha(m, K) \int_{m \cdot K} g(x) d \mu_{m \cdot K} x=\int_{K} g(m \cdot x) d \mu_{K} x
$$

$\left(g \in C_{0}(G)\right.$ ) shows that $\alpha$ is continuous on $M \times \mathscr{K}(G)$. Thus, for each $m$, the function $V^{(m)}$, initially defined on $C_{0}(Y)$ by

$$
V^{(m)}(f)(m \cdot K, m \cdot x)=\alpha(m, K) f(K, x),
$$

extends to a *-isomorphism of $C_{s}^{*}(G)$ onto itself; and we have $W^{\beta_{m}\left(\left\langle K, T_{\rangle}\right)\right.}=W^{K, T} \circ V^{\left(m^{-1}\right)}$. Now $m \rightarrow V^{\left(m^{-1}\right)}$ is continuous in the topology of pointwise convergence on $C_{s}^{*}(G)$. Thus an appeal to Proposition 1.6 completes the proof.

Lemma 2.8. Every irreducible *-representation $S$ of $C_{s}^{*}(G)$ is of the form $W^{K, T}$ for some unique $\langle K, T\rangle$ in $\mathscr{S}(G)$.

Proof. It follows from $[19, \S 26]$ that Kernel $(S)$ must contain $\left\{f \in C_{0}(Y) \mid f_{K}=0\right\}$ for some $K$ in $\mathscr{K}(G)$. Thus $S$ is lifted from some $K$. The uniqueness is evident.

For each $K$ in $\mathscr{K}(G)$, let us define $\hat{A}_{K}=\left\{W^{K, T} \mid T \in \hat{K}\right\}$. It follows from Lemmas 2.5 and 2.8 that the $\hat{A}_{K}$ are pairwise disjoint nonvoid closed subsets of $C_{s}^{*}(G)^{\wedge}$ whose union is $C_{s}^{*}(G)^{\wedge}$.

The following new topology of $\mathscr{K}(G)$ is intimately related to the openness of the mapping $\pi$.

DEFINITION. The representational topology of $\mathscr{K}(G)$ is that topology which makes the mapping $K \rightarrow \hat{A}_{K}$ a homeomorphism with respect to the compactopen topology of closed subsets of $C_{s}^{*}(G)^{\wedge}$.

There is another description of the compact-open and the representational topologies, in terms of norms. We shall denote by \|\|$_{c}$ not only the norm of $C_{s}^{*}(G)$ but the norm of each $C^{*}(K)$ as well.

LEMMA 2.9. $K_{i} \rightarrow K$ in $\mathscr{K}(G)$ (in the compact-open topology) if and only if

$$
\underset{i}{\limsup }\left\|f_{K_{i}}\right\|_{c} \leqq\left\|f_{K}\right\|_{c}
$$

for all $f$ in $A_{s}(G)$.

Proof. Assume that $K_{i} \rightarrow K$. By the compactness of $\mathscr{X}\left(C_{s}^{*}(G)^{\wedge}\right)$ every subnet of $\left\{K_{i}\right\}$ has a subnet $\left\{K_{j}^{\prime}\right\}$ such that $\hat{A}_{K_{j}^{\prime}} \rightarrow Q$ in the compact-open topology of $\mathscr{X}\left(C_{s}^{*}(G)^{\wedge}\right)$. By Lemma $2.5 Q \subset \hat{A}_{K}$. But by Theorem 2.2 of [6], $\lim _{j}\left\|f_{K_{j}^{\prime}}\right\|_{c}=\sup _{S \in Q}\left\|S_{f}\right\| ;$ hence

$$
\lim _{j}\left\|f_{K_{j}^{\prime}}\right\|_{c} \leqq \sup _{S \in \hat{A}_{K}}\left\|S_{f}\right\|=\left\|f_{K}\right\|_{c} \text { for } f \in A_{s}(G) .
$$

By the arbitrariness in the definition of the subnet $\left\{K_{j}^{\prime}\right\}$, this implies (1). The converse is evident. 
Lemma 2.10. The representational topology of $\mathscr{K}(G)$ is the smallest topology of $\mathscr{K}(G)$ making all the "norm-functions" $K \rightarrow\left\|f_{K}\right\|_{c}\left(f \in A_{s}(G)\right)$ continuous.

Proof. Theorem 2.2 of [6].

LEMmA 2.11. The representational topology contains the compact-open topology of $\mathscr{K}(G)$.

Proof. Lemmas 2.9 and 2.10.

We do not know at present whether the representational topology can ever be different from the compact-open topology of $\mathscr{K}(G)$.

Lemma 2.12. The map $\pi:\langle K, T\rangle \rightarrow K$ is open on $\mathscr{S}(G)-\{\theta\}$ onto $\mathscr{K}(G)$ with respect to the inner hull-kernel topology of the former and the representational topology of the latter.

Proof. Let $K$ be in the representational closure of a subset $\mathscr{L}$ of $\mathscr{K}(G)$, and let $T$ be a nonzero representation of $K$. For each $L$ in $\mathscr{L}$ let $S(L)$ be a unitary representation of $L$ whose spectrum is all of $\hat{L}$. Then, if $\left\{L^{i}\right\}$ is a net of elements of $\mathscr{L}$ converging representationally to $K$, it follows from the definition of the inner hull-kernel topology (and the fact that $\operatorname{Sp}\left(W^{K, T}\right) \subset \hat{A}_{K}$ ) that $\left\langle L^{i}, S\left(L^{i}\right)\right\rangle \rightarrow\langle K, T\rangle$ in $\mathscr{S}(G)$. But this means that $\pi$ is open.

The continuity of $\pi$ with respect to the representational topology of $\mathscr{K}(G)$, like the openness of $\pi$ with respect to the compact-open topology of $\mathscr{K}(G)$, is questionable. We now show that the two questions are equivalent.

THEOREM 2.1. For each $K$ in $\mathscr{K}(G)$ the following conditions are equivalent:

(i) The representational neighborhoods and the compact-open neighborhoods of $K$ are the same;

(ii) For each $f$ in $A_{s}(G)$, the map $L \rightarrow\left\|f_{L}\right\|_{c}$ is continuous at $K$ in the compactopen topology;

(iii) $\pi$ is continuous at $\langle K, T\rangle$ (with respect to the representational topology of $\mathscr{K}(G)$ ) for each nonzero unitary representation $T$ of $K$;

(iv) $\pi$ is open (18) at $\langle K, T\rangle$ (with respect to the compact-open topology of $\mathscr{K}(G))$ for each nonzero unitary representation $T$ of $K$.

Proof. (i) and (ii) are equivalent by Lemma 2.10. (i) implies (iii) by Lemma 2.5; (i) implies (iv) by Lemma 2.12 .

Assume (iii). Let $K^{i} \rightarrow K$ in the compact-open topology of $\mathscr{K}(G)$. Suppose that $T$ is some nonzero unitary representation of $G$. For each $f$ in $C_{0}(Y)$ and each $\xi, \eta$ in $H(T)$, Lemma 2.1 gives

$$
\lim _{i}\left(W^{K^{i}, T \mid K^{l}}(f) \xi, \eta\right)=\left(W^{K, T \mid K}(f) \xi, \eta\right) .
$$

(18) A map $f$ is open at a point $x$ if it carries each neighborhood of $x$ onto a neighborhood of $f(x)$. 
It follows that $\liminf _{i}\left\|\left(T \mid K^{i}\right)\left(f_{K^{i}}\right)\right\| \geqq\left\|(T \mid K)\left(f_{K}\right)\right\|$. Thus, by Proposition 1.4, $\left\langle K^{i}, T \mid K^{i}\right\rangle \rightarrow\langle K, T \mid K\rangle$ in $\mathscr{S}(G)$. By (iii) this implies that $K^{i} \rightarrow K$ representationally. So compact-open convergence to $K$ implies representational convergence. This and Lemma 2.11 give (i). Hence (iii) implies (i).

Assume (iv). Let $K^{i} \rightarrow K$ in the compact-open topology. By the compactness of $\mathscr{X}\left(C_{s}^{*}(G)^{\wedge}\right)$ we may replace $\left\{K^{i}\right\}$ by a subnet and assume $\hat{A}_{K^{i}} \rightarrow$ some $\mathscr{Y}$ in $\mathscr{X}\left(C_{s}^{*}(G)^{\wedge}\right)$. By Lemma $2.5 \mathscr{Y} \subset \hat{A}_{K}$. Assume $W^{K, T} \in \hat{A}_{K}-\mathscr{Y}$. Since $\mathscr{Y}$ is closed there is a compact $\left({ }^{19}\right)$ neighborhood $V$ of $W^{K, T}$ in $C_{s}^{*}(G)^{\wedge}$ which does not intersect $\mathscr{Y}$. Hence $\hat{A}_{K^{i}} \cap V=\Lambda i$-eventually. On the other hand, by the assumed openness of $\pi, U=\pi(\{S \mid \mathrm{Sp}(S)$ intersects $V\})$ is a compact-open neighborhood of $K$, so that $K_{i} \in U i$-eventually; this implies that, $i$-eventually, $\hat{A}_{K^{\prime}}$ intersects $V$. Thus we have a contradiction. So $\mathscr{Y}=\hat{A}_{K}$; whence $\hat{A}_{K^{t}} \rightarrow \hat{A}_{K}$ in $\mathscr{X}\left(C_{s}^{*}(G)^{\wedge}\right)$; that is, $K^{i} \rightarrow K$ representationally. We have now shown that every net converging to $K$ in the compact-open topology has a subnet converging to $K$ representationally. This, together with Lemma 2.11, gives (i). So (iv) implies (i).

Definition. If any of the conditions (i)-(iv) of Theorem 2.1 hold, we shall say that the subgroup representation theory (of $G$ ) is continuous at $K$.

THEOREM 2.2. A sufficient condition for the subgroup representation theory to be continuous at $K$ is that every irreducible unitary representation $T$ of $K$ be weakly contained in $S \mid K$ for some unitary representation $S$ of $G$.

Proof. Assume the condition. Given $f$ in $C_{0}(Y)$ and $T$ in $R$, choose $S$ as in the condition. Then $\left\|(S \mid K)\left(f_{K}\right)\right\| \geqq\left\|T\left(f_{K}\right)\right\|$. Hence, if $K_{i} \rightarrow K$ in the compactopen topology of $\mathscr{K}(G)$, the argument of the implication (iii) $\Rightarrow$ (i) in the proof of Theorem 2.1 gives

$$
\begin{aligned}
\liminf _{i}\left\|f_{K_{i}}\right\|_{c} & \geqq \underset{i}{\liminf }\left\|\left(S \mid K_{i}\right)\left(f_{K_{i}}\right)\right\| \\
& \geqq\left\|(S \mid K)\left(f_{K}\right)\right\| \geqq\left\|T\left(f_{K}\right)\right\| .
\end{aligned}
$$

This is true for all $T$ in $\hat{K}$. Hence

$$
\underset{i}{\liminf }\left\|f_{K_{i}}\right\|_{c} \geqq \sup _{T \in \hat{K}}\left\|T\left(f_{K}\right)\right\|_{c}=\left\|f_{K}\right\|_{c} .
$$

This together with Lemma 2.9 proves condition (ii) of Theorem 2.1.

We shall observe in $\$ 5$ (Theorem 5.1 and Proposition 5.2) that the condition of Theorem 2.2 is always satisfied if $G$ is compact, commutative or discrete. So we have:

COROLlaRY. The representational and compact-open topologies of $\mathscr{K}(G)$ coincide whenever $G$ is either compact, commutative, or discrete.

(19) Recall that $C_{8}^{*}(G)^{\wedge}$ is locally compact [6, Theorem 2.1$]$. 
In $\S 6$ we shall give an example of the failure of the condition of Theorem 2.2. For another consequence of Theorem 2.2 see $\$ 5$, Corollary 6 .

3. The topology of $\mathscr{S}(G)$ in terms of functions of positive type. It is a now classical result that the weak* topology of characters on a locally compact Abeiian group coincides with the topology of uniform convergence on compact sets. In [5, Theorem 2.2], we generalized this result to describe the inner hull-kernel topology of unitary representations of an arbitra1y locally compact group $G$. In this section we shall generalize it still further, to describe the inner hull-kernel topology of subgroup representations of $G$.

The first step is to define a topology for $\bigcup_{K \in \mathscr{X}(G)} C(K)$, so that a function defined on $K$ is allowed to approach a function defined on $K_{0}$ as $K$ approaches $K_{0}$. This will be done in such a way that, for functions having a fixed domain, the topology is that of uniform convergence on compact sets.

Let $X$ be an arbitrary locally compact Hausdorff space, $Z$ the complex number system, and $S=X \times Z$. A subset of $S$ is semicompact if it is closed and its projection on $X$ has compact closure; $\mathscr{D}$ will be the family of all semicompact subsets of $S$. As in $\S 1 \mathscr{X}(S)$ is the family of all closed subsets of $S$. Let $U(D ; \mathscr{F})=\{A \in \mathscr{X}(S) \mid A \cap D=\Lambda, A \cap B \neq \Lambda$ for each $B$ in $\mathscr{F}\}$. The semicompactopen topology of $\mathscr{X}(S)$ will be that topology in which the $U(D ; \mathscr{F})$, where $D \in \mathscr{D}$ and $\mathscr{F}$ is a finite family of nonvoid open subsets of $S$, form a basis of open sets.

Let $\mathscr{E}(X)$ be the union of all the spaces $C(A)$, where $A \in \mathscr{X}(S)$; thus the elements of $\mathscr{E}(X)$ are continuous complex functions $f$ such that $D(f) \in \mathscr{X}(S)$. Identifying a function with its graph we have $\mathscr{E}(X) \subset \mathscr{X}(S)$. We shall always consider $\mathscr{E}(X)$ as equipped with the (relativized) semicompact-open topology.

LemMA 3.1. The map $f \rightarrow D(f)$ of $\mathscr{E}(X)$ onto $\mathscr{X}(X)$ is continuous and open.

Proof. If $C$ is a compact subset of $X$ and $\mathscr{F}$ is a finite family of open subsets of $X$, the inverse image of $U(C ; \mathscr{F})$ under the above map is the open set $U(C \times Z ;\{A \times Z \mid A \in \mathscr{F}\})$. So the map is continuous.

Suppose $\mathscr{Y} \subset \mathscr{X}(X), Y \in \overline{\mathscr{Y}}, f \in \mathscr{E}(X)$, and $D(f)=Y$. To prove openness, it suffices to approximate $f$ in $\mathscr{E}(X)$ by elements $g$ for which $D(g) \in \mathscr{Y}$. Choose a neighborhood $U(D ; \mathscr{F})$ of $f$ where $D \in \mathscr{D}$ and $\mathscr{F}$ is a finite family of open sets with compact closure. Let $E$ be a compact subset of $X$ containing the $X$, rojections of $D$ and of all $A$ in $\mathscr{F}$. By Tietze's theorem there is an $f_{0}$ in $C(X)$ coinciding with $f$ on $Y \cap E$. Now let $Y_{i} \rightarrow Y$ in $\mathscr{X}(X)$, where $Y_{i} \in \mathscr{Y}$. It will be sufficient to show that

$$
f_{0} \mid Y_{i} \in U(D ; \mathscr{F}) \text { i-eventually. }
$$

If it were false that

$$
\left(f_{0} \mid Y_{i}\right) \cap D=\Lambda \text { i-eventually, }
$$


we could pass to a subnet and choose for each $i$ an $x_{i}$ in $Y_{i} \cap E$ with $\left\langle x_{i}, f_{0}\left(x_{i}\right)\right\rangle \in D$. By the compactness of $E$ we again pass to a subnet and assume $x_{i} \rightarrow x \in E \cap Y$. Since $f_{0}$ is continuous, $\left\langle x_{i}, f_{0}\left(x_{i}\right)\right\rangle \rightarrow\left\langle x, f_{0}(x)\right\rangle \in D$, contradicting $f \cap D=\Lambda$. So (2) holds. Again, if $A \in \mathscr{F}$, choose an $x_{0}$ in $Y$ and an open $X$-neighborhood $V$ of $x_{0}$ so that $\left\langle x, f_{0}(x)\right\rangle \in A$ for all $x$ in $V$. Since $Y_{i} \rightarrow Y, Y_{i} i$-eventually intersects $V$, so that

$$
f_{0} \mid Y_{i} \text { i-eventually intersects } A \text {. }
$$

Now (2) and (3) imply (1).

LEMMA 3.2. Suppose $f \in \mathscr{E}(X),\left\{f_{i}\right\}$ is a net of elements of $\mathscr{E}(X)$, and $D\left(f_{i}\right) \rightarrow D(f)$ in $\mathscr{X}(X)$. Then $f_{i} \rightarrow f$ in $\mathscr{E}(X)$ if and only if, for each subnet $\left\{f_{j}^{\prime}\right\}$ of $\left\{f_{i}\right\}$ and each choice of $x_{j}$ in $D\left(f_{j}^{\prime}\right)$ (for each $\left.j\right)$ such that $x_{j} \rightarrow$ some $x$ in $D(f)$, we have $f_{j}^{\prime}\left(x_{j}\right) \rightarrow f(x)$.

Proof. (I) Assume $f_{i} \rightarrow f$, and let $\left\{f_{j}^{\prime}\right\},\left\{x_{j}\right\}, x$ be as above; let $\varepsilon>0$. If $V$ is a compact $X$-neighborhood of $x$ such that $|f(y)-f(x)|<\varepsilon$ for $y \in V \cap D(f)$, the set $D=\{\langle y, \lambda\rangle \in S|y \in V,| \lambda-f(x) \mid \geqq \varepsilon\}$ is semicompact and $f \cap D=\Lambda$. So $f_{j}^{\prime} \cap D=\Lambda j$-eventually. But $x_{j} \in V$ j-eventually, so that $\left|f_{j}^{\prime}\left(x_{j}\right)-f(x)\right|<\varepsilon$ $j$-eventually. Thus $f_{j}^{\prime}\left(x_{j}\right) \rightarrow f(x)$.

(II) Assume the condition of the lemma. If $D \in \mathscr{D}, f \cap D=\Lambda$, and it is false that

$$
f_{i} \cap D=\Lambda \text { i-eventually, }
$$

then, passing twice to a subnet, we may choose for each $i$ an $\left\langle x_{i}, f_{i}\left(x_{i}\right)\right\rangle$ in $f_{i} \cap D$ such that $x_{i} \rightarrow x \in D(f)$. By assumption this implies $f_{l}\left(x_{i}\right) \rightarrow f(x)$, whence $\langle x, f(x)\rangle \in D$, which is impossible. So (4) holds whenever $D \in \mathscr{D}, f \cap D=\Lambda$. Again, let $A$ be an open subset of $S$ intersecting $f$ in some point $\langle x, f(x)\rangle$. Since $x \in D(f)$ and $D\left(f_{i}\right) \rightarrow D(f)$, there is a subnet $\left\{f_{j}^{\prime}\right\}$ of $\left\{f_{i}\right\}$, and an $x_{j}$ in $D\left(f_{j}^{\prime}\right)$ for each $j$, such that $x_{j} \rightarrow x$. By the assumption, $\left\langle x_{j}, f_{j}^{\prime}\left(x_{j}\right)\right\rangle \rightarrow\langle x, f(x)\rangle$; so that $f_{j}^{\prime} j$-eventually intersects $A$. Since this is true on replacing $\left\{f_{i}\right\}$ by any subnet, we conclude that

$$
f_{i} \cap A \neq \Lambda \text { i-eventually. }
$$

Now (4) and (5) show that $f_{i} \rightarrow f$.

Applying Lemma 3.2 to the case that all $f_{i}$ have the same domain $Y$, we get:

LEMMA 3.3. If $Y \in \mathscr{X}(X)$, the semicompact-open topology of $\mathscr{E}(X)$, relativized to $\{f \mid D(f)=Y\}$, is just the topology of uniform convergence on compact subsets of $Y$.

The following easy corollary of Lemma 3.2 expresses the continuity of restriction: 
Lemma 3.4. Let $\mathscr{W}=\{\langle Y, f\rangle \mid Y \in \mathscr{X}(X), f \in \mathscr{E}(X), \quad Y \subset D(f)\}$. Then the map $\langle Y, f\rangle \rightarrow f \mid Y$ is continuous on $\mathscr{W}$ to $\mathscr{E}(X)$ (the topology of $\mathscr{W}$ being relativized from the product $\mathscr{X}(X) \times \mathscr{E}(X)$ ).

We now return to our locally compact group $G$ with unit $e$, and with smooth choice of Haar measures $\left\{\mu_{K}\right\}$ on $\mathscr{K}(G)$. We denote by $\mathscr{E}_{s}(G)$ the topological subspace of $\mathscr{E}(G)$ consisting of those $f$ for which $D(f) \in \mathscr{K}(G)$. Let

$$
Y=\{\langle K, x\rangle \mid K \in \mathscr{K}(G), x \in K\}
$$

as before.

Proposition 3.1. Let $C$ be a compact subset of $Y$, and $W$ the set of those $f$ in $C_{0}(Y)$ whose supports are contained in $C$; we equip $W$ with the topology of uniform convergence on $Y$. Then the map

$$
\langle f, g\rangle \rightarrow \int_{D(f)} f(x) g(D(f), x) d \mu_{D(f)} x
$$

is continuous on $\mathscr{E}_{s}(G) \times W$.

Proof. Let $f_{i} \rightarrow f$ in $\mathscr{E}_{s}(G)$ and $g_{i} \rightarrow g$ in $W$; and let $F$ be such an element of $C_{0}(Y)$ that $F(D(f), x)=f(x)$ for all $x$ such that $\langle D(f), x\rangle \in C$. Let $K=D(f), K_{i}=D\left(f_{i}\right)$. Since $K_{i} \rightarrow K$ (Lemma 3.1), Lemma 2.1 gives

$$
\lim _{i} \int_{K_{i}} F\left(K_{i}, x\right) g\left(K_{i}, x\right) d \mu_{K_{i}} x=\int_{K} f(x) g(K, x) d \mu_{K} x .
$$

I claim that, for each $\varepsilon>0$, we have $i$-eventually

$$
\left|f_{i}(x)-F(K, x)\right|<\varepsilon \text { whenever }\left\langle K_{i}, x\right\rangle \in C .
$$

Indeed, otherwise, passing to a subnet we could find an $\varepsilon>0$ and an $x_{i}$ for each $i$, such that $\left\langle K_{i}, x_{i}\right\rangle \in C,\left|f_{i}\left(x_{i}\right)-F\left(K_{i}, x_{i}\right)\right| \geqq \varepsilon$. By the compactness of $C$ we may even assume $x_{i} \rightarrow x \in K$. But then by Lemma $3.2 f_{i}\left(x_{i}\right) \rightarrow f(x)$. This and the fact that $F\left(K_{i}, x_{i}\right) \rightarrow F(K, x)=f(x)$ contradict the definition of $x_{i}$. So (8) holds. By Lemma 1.1 of [11] there is a constant $k$ with $\mu_{K_{i}}\left(\left\{x \mid\left\langle K_{i}, x\right\rangle \in C\right\}\right)<k$ for all $i$. So, by (8), for each $\varepsilon>0$ we have $i$-eventually

$$
\begin{array}{rl}
\mid \int_{K} f_{i}(x) g_{i}\left(K_{i}, x\right) d \mu_{K_{i}} x-\int_{K_{i}} & F\left(K_{i}, x\right) g\left(K_{i}, x\right) d \mu_{K_{i}} x \mid \\
\leqq & \int_{K_{i}}\left|f_{i}(x)-F\left(K_{i}, x\right)\right|\left|g_{i}\left(K_{i}, x\right)\right| d \mu_{K_{i}} x \\
& +\int_{K_{i}}\left|F\left(K_{i}, x\right)\right|\left|g_{i}\left(K_{i}, x\right)-g\left(K_{i}, x\right)\right| d \mu_{K_{i}} x \\
& \leqq \\
\qquad g_{i}\left\|_{\infty} k \varepsilon+\right\| F\left\|_{\infty}\right\| g_{i}-g \|_{\infty} k .
\end{array}
$$

This combined with (7) gives (6). 
Definition. An element $\phi$ of $\mathscr{E}_{s}(G)$ is of positive type, and associated with the subgroup representation $\langle K, T\rangle$ if $D(\phi)=K$ and there exists a vector $\xi$ in $H(T)$ such that $\phi(x)=\left(T_{x} \xi, \xi\right)$ for all $x$ in $K$. If $Q \subset \mathscr{S}(G), \phi$ is associated with $Q$ if it is associated with some $\langle K, T\rangle$ in $Q$.

THEOREM 3.1. Let $\langle K, T\rangle$ be a subgroup representation of $G$. If $\phi_{1}, \cdots, \phi_{n}$ is a finite sequence of functions of positive type on $K$ associated with $\langle K, T\rangle$, and if for each $r=1, \cdots, n V_{r}$ is an open neighborhood of $\phi_{r}$ (in the semicompactopen topology of $\left.\mathscr{E}_{s}(G)\right)$, we define $U=U\left(\phi_{1}, \cdots, \phi_{n} ; V_{1}, \cdots, V_{n}\right)$ to be the set of all $\left\langle K^{\prime}, T^{\prime}\right\rangle$ in $\mathscr{S}(G)$ for which there exist $\phi_{1}^{\prime}, \cdots, \phi_{n}^{\prime}$ such that, for each $i$,

(i) $\phi_{i}^{\prime} \in V_{i}$,

(ii) $D\left(\phi_{i}^{\prime}\right)=K^{\prime}$,

(iii) $\phi_{i}^{\prime}$ is a finite sum of functions of positive type associated with $T^{\prime}$.

Then the set of all such $U$ forms a basis of neighborhoods of $\langle K, T\rangle$ in the inner hull-kernel topology of $\mathscr{S}(G)$.

We preface the proof with the following lemma:

Lemma 3.5. Let $f \in C_{0}(Y)$, and let $F$ be some extension of $f$ to an element of $\left.C_{0}(\mathscr{K}(G) \times G)\right)$. For each $x$ in $G$ put $F_{x}(K, y)=F\left(K, x^{-1} y\right)(K \in \mathscr{K}(G), y \in G)$, and define $f_{x}=F_{x} \mid Y$. Then $f_{x} \in C_{0}(Y)$ for each $x$; and the map $x \rightarrow f_{x}$ is continuous on $G$ to $C_{0}(Y)$ (in the $A_{s}(G)$-norm of the latter, see \$2).

Proof. That $f_{x} \in C_{0}(Y)$ is obvious. Fix $x_{0}$ in $G$ and $\varepsilon>0$. Let $W$ be a compact subset of $G$ such that the interior of $\mathscr{K}(G) \times W$ contains the compact support of $F$. Then there is a neighborhood $V$ of $x_{0}$ such that (i) $\mathscr{K}(G) \times x_{0} W$ contains the compact support of $F_{x}$ for each $x$ in $V$, and (ii) $\left\|F_{x}-F_{x_{0}}\right\|_{\infty}<\varepsilon$ for all $x$ in $V$. By Lemma 1.1 of [11] there is a constant $k$ such that $\mu_{K}(W \cap K)<k$ for all $K$. Thus, for all $x$ in $V$,

$$
\left\|f_{x}-f_{x_{0}}\right\|_{A_{-}(G)}=\sup _{K \in \mathscr{X}(G)} \int_{K}\left|F\left(K, x^{-1} y\right)-F\left(K, x_{0}^{-1} y\right)\right| d \mu_{K} y<k \varepsilon .
$$

This proves the lemma.

Proof of theorem. (I) Let $\phi_{1}, \cdots, \phi_{n}, V_{1}, \cdots, V_{n}$ be as in the definition of $U$; without loss of generality suppose $\phi_{r}(e)=1$. Fix a subset $Q$ of $\mathscr{S}(G)$ with $\langle K, T\rangle$ in its closure. To show that $U$ is a neighborhood of $\langle K, T\rangle$ it will suftice to show that $Q$ intersects $U$.

Choose $\xi_{1}, \cdots, \xi_{n}$ in $H(T)$ so that $\phi_{r}(x)=\left(T_{x} \xi_{r}, \xi_{r}\right) \quad(x \in K)$; since $\phi_{r}(e)=1$, $\left\|\xi_{r}\right\|=1$. By $\left[5\right.$, Theorem 2.1], applied to $C_{s}^{*}(G)$, there is a net $\left\{\left\langle K^{i}, T^{i}\right\rangle\right\}$ of elements of $Q$, and for each $i$ and each $r=1, \cdots, n$ a sum $\phi_{r}^{i}$ of positive functionals on $C_{s}^{*}(G)$ associated with $W^{K^{i}, T^{i}}$, such that

$$
\left\|\phi_{r}^{i}\right\| \leqq 1 \text { for all } i \text { and } r
$$

and 


$$
\phi_{r}^{i} \underset{i}{\rightarrow} \phi_{r} \text { pointwise }\left({ }^{20}\right) \text { on } C_{s}^{*}(G) \text {. }
$$

We now fix an $\varepsilon$ with $1>\varepsilon>0$ so that, for each $r, \chi \in V_{r}$ whenever $\chi \in C(K)$ and $\left|\chi(x)-\phi_{r}(x)\right|<\varepsilon$ for all $x$ in $K$. Next we choose an $f$ in $C_{0}(Y)$ so that $\left\|T_{f} \xi_{r}-\xi_{r}\right\|<\varepsilon / 3$ for each $r$. Then

$$
\left|\left(T_{x} T_{f} \xi_{r}, T_{f} \xi_{r}\right)-\phi_{r}(x)\right|<\varepsilon
$$

For $x$ in $K$ we verify that $\left(T_{x} T_{f} \xi_{r}, T_{f} \xi_{r}\right)=\phi_{r}\left(f^{*} * f_{x}\right)$, where, for each $x$ in $G$, $f_{x}$ is to mean the same as in Lemma 3.5. Applying Gelfand's Lemma to (9) and (10) and using Lemma 3.5, we find that for each $r$,

$$
\phi_{r}^{i}\left(f^{*} * f_{x}\right) \underset{i}{\rightarrow} \phi_{r}\left(f^{*} * f_{x}\right)
$$

uniformly in $x$ on compact subsets of $G$. Now let $\chi_{r}^{i}, \chi_{r}$ be the elements of $\mathscr{E}_{s}(G)$ defined by: $D\left(\chi_{r}^{i}\right)=K_{i}, D\left(\chi_{r}\right)=K, \chi_{r}^{i}(x)=\phi_{r}^{i}\left(f^{*} * f_{x}\right)\left(x \in K_{i}\right), \chi_{r}(x)=\phi_{r}\left(f^{*} * f_{x}\right)$ $(x \in K)$. From the corresponding property of $\phi_{r}^{i}$ we verify that $\chi_{r}^{i}$ is a sum of functions of positive type associated with $T^{i}$. Further, by (11) and the definition of $\varepsilon$,

$$
\chi_{r} \in V_{r} \text { for each } r \text {. }
$$

Now it follows from (10) that $K^{i} \rightarrow K$ in $\mathscr{K}(G)$. If we had $x_{i} \in K^{i}$ for each $i$ and $x_{i} \rightarrow x \in K$, it would follow from (12) that $\chi_{r}^{i}\left(x_{i}\right) \rightarrow \chi_{r}(x)$. Hence by Lemma $3.2 \chi_{r}^{i} \rightarrow \chi_{r}$ in $\mathscr{E}_{s}(G)$. This and (13) show that $Q$ intersects $U$. So $U$ is an inner hull-kernel neighborhood of $\langle K, T\rangle$.

(II) To show that the $U$ of the theorem form a basis of neighborhoods of $\langle K, T\rangle$, it suffices to take a net $\left\{\left\langle K^{i}, T^{i}\right\rangle\right\}$ of elements of $\mathscr{S}(G)$ which is eventually inside each such $U$, and to show that some subnet of it converges to $\langle K, T\rangle$.

Fix functions $\phi_{1}, \cdots, \phi_{n}$ of positive type on $K$ associated with $T$. By the assumption on $\left\{\left\langle K^{i}, T^{i}\right\rangle\right\}$, we can replace it by a subnet, and then for each $i$ and $r=1, \cdots, n$ choose a $\phi_{r}^{i}$ such that: (a) $\phi_{r}^{i}$ is a sum of functions of positive type on $K_{i}$ associated with $T^{i}$, and (b) $\phi_{r}^{i} \rightarrow \phi_{r}$ in $\mathscr{E}_{s}(G)$ for each $r$. But then the $\phi_{r}^{i}$ are bounded in norm uniformly in $i$ and $r$; and for each $r$ and each $g$ in $C_{0}(Y)$ we have by Proposition 3.1:

$$
\begin{aligned}
\lim _{i} \phi_{r}^{i}(g) & =\lim _{i} \int_{K^{i}} \phi_{r}^{i}(x) g\left(K^{i}, x\right) d \mu_{K^{i}} x \\
& =\int_{K} \phi_{r}(x) g(K, x) d \mu_{K^{\prime}} x=\phi_{r}(g) .
\end{aligned}
$$

Thus, by Theorem 2.1 of $[5],\left\langle K^{i}, T^{i}\right\rangle \rightarrow\langle K, T\rangle$.

The preceding theorem can be rephrased as follows:

(20) We identify functions of positive type on a closed subgroup $K$ with the corresponding positive functionals on $C_{s}^{*}(G)$. 
THEOREM 3.1'. Let $\left\{\left\langle K^{i}, T^{i}\right\rangle\right\}$ be a net of elements of $\mathscr{S}(G)$ and $\langle K, T\rangle$ an element of $\mathscr{S}(G)$. Then $\left\langle K^{i}, T^{i}\right\rangle \rightarrow\langle K, T\rangle$ if and only if, for each finite sequence $\phi_{1}, \cdots, \phi_{n}$ of functions of positive type on $K$ associated with $\langle K, T\rangle$, and each subnet of $\left\{\left\langle K^{i}, T^{i}\right\rangle\right\}$, there exist (i) a subnet $\left\{\left\langle K^{\prime j}, T^{\prime j}\right\rangle\right\}$ of that subnet, and (ii) for each $j$ and each $r=1, \cdots, n$ a finite sum $\phi_{r}^{j}$ of functions of positive type associated with $\left\langle K^{\prime j}, T^{\prime j}\right\rangle$, such that $\phi_{r}^{i} \rightarrow \phi_{r}$ in $\mathscr{E}_{s}(G)$ for each $r$.

REMARK. In Theorem 3.1 assume that $T$ is irreducible. Then the theorem will still be true if in (iii) of its statement we replace "a finite sum of functions of positive type" by "a function of positive type." Similarly for Theorem 3.1'. The argument verifying this is the same as for the similar remark following Theorem 2.1 of [5].

Combining Theorem 3.1' with Lemma 3.4, we obtain the continuity of the operation of restricting a subgroup representation to a smaller subgroup:

THeOREM 3.2. Let $\mathscr{W}=\{\langle H, K, T\rangle \mid\langle K, T\rangle \in \mathscr{S}(G), \quad H \in \mathscr{K}(G), \quad H \subset K\}$; and give to $\mathscr{W}$ the topology relativized from the product $\mathscr{K}(G) \times \mathscr{S}(G)$. Then the map $\langle H, K, T\rangle \rightarrow\langle H, T \mid H\rangle$ is continuous on $\mathscr{W}$ to $\mathscr{S}(G)$.

THEOREM 3.3. Let $Q$ be a subset of $\mathscr{S}(G)$ and $\langle K, T\rangle$ an element of $\mathscr{S}(G)$ weakly contained in $Q$. Let $L$ be a closed subgroup of $G$ such that $L \subset K$ and $L \subset K^{\prime}$ for all $\left\langle K^{\prime}, T^{\prime}\right\rangle$ in $Q$. Then $T \mid L$ is weakly contained in the family of all $T^{\prime} \mid L$, where $\left\langle K^{\prime}, T^{\prime}\right\rangle$ ranges over $Q$.

Proof. Each element $S$ of $\mathrm{Sp}(T)$ (considered as a subset of $\hat{A}_{K}$ ) is weakly contained in $Q$, and hence belongs to the closure of $Q$ (Proposition 1.1). So by Theorem $3.2 S \mid L$ belongs to the closure of, and hence is weakly contained in, the set of all restrictions to $L$ of elements of $Q$. But, by [4, p. 371, Corollary], $T \mid L$ is weakly equivalent to $\{S|L| S \in \mathrm{Sp}(T)\}$. Combining these facts we obtain the theorem.

4. The continuity of the inducing operation. The theory of induced representations was first systematically developed for separable locally compact groups by Mackey in [14]. An equivalent treatment, more convenient in the nonseparable situation, was given by Blattner in $[2 ; 3]$. We state here for the reader's convenience some facts proved in [3].

As usual, $G$ is a locally compact group with unit $e$, and $\left\{\mu_{K}\right\}$ is a smooth choice of left Haar measures on $\mathscr{K}(G)$; we write $\mu$ for $\mu_{G} . \Delta_{K}$ is the modular function on $K$; and we write $\Delta$ for $\Delta_{G}$.

A complex-valued regular Borel measure $\rho$ on $G$ is of positive type on $G$ if

$$
\int_{G}\left(f^{*} * f\right)(x) d \rho x \geqq 0
$$


for all $f$ in $C_{0}(G)$. If $d \rho x=\phi(x) d \mu x$, where $\phi \in C(G)$, then $\rho$ is of positive type on $G$ if and only if $\phi$ is of positive type in the usual sense. Each measure $\rho$ of positive type on $G$ generates a unitary representation $T^{\rho}$ of $G$ as follows: Let $(f, g)_{\rho}=\int_{G}\left(g^{*} * f\right)(x) d \rho x$ for $f, g \in C_{0}(G)$. Then $N=\left\{f \in C_{0}(G) \mid(f, f)_{\rho}=0\right\}$ is a linear subspace of $C_{0}(G)$; and $($,$) induces an inner product, also called$ $($,$) , on C_{0}(G) / N$. If $\Phi=\Phi_{G, \rho}$ is the natural map of $C_{0}(G)$ into the completion $H_{\rho}$ of $C_{0}(G) / N$ under $($,$) then the relation$

$$
T_{x}^{\rho}(\Phi(f))=\Phi\left(f_{x}\right) \quad\left(f \in C_{0}(G), x \in G\right)
$$

(where $f_{x}(y)=f\left(x^{-1} y\right)$ ) defines a unitary representation $T^{\rho}$ of $G$ on $H_{\rho}$. If $f, h \in C_{0}(G)$, then $T_{h}^{\rho}(\Phi(f))=\Phi(h * f)$.

The following result is proved in [3]:

THEOREM 4.1. Let $K$ be a closed subgroup of $G$, and $\sigma$ a complex regular measure of positive type on $K$. Let $d \rho x=\left(\Delta(x) / \Delta_{K}(x)^{1 / 2} d \sigma x\right.$. Then $\rho$, considered as a complex measure on $G$ (carried by $K)$, is of positive type on $G$. Further, $T^{\rho}$ is unitarily equivalent to the representation of $G$ induced from the representation $T^{\sigma}$ of $K$.

We now prove our main theorem:

THEOREM 4.2. Let $\mathscr{W}=\{\langle H, K, T\rangle \mid\langle K, T\rangle \in \mathscr{S}(G), \quad H \in \mathscr{K}(G), H \supset K\}$; and give to $\mathscr{W}$ the topology relativized from the product $\mathscr{K}(G) \times \mathscr{S}(G)$. Then the map $\langle H, K, T\rangle \rightarrow\left\langle H,{ }_{H} U^{T}\right\rangle$ is continuous on $\mathscr{W}$ to $\mathscr{S}(G)$.

Proof. We shall suppose that $H^{i} \rightarrow H$ in $\mathscr{K}(G)$ and $\left\langle K^{i}, S^{i}\right\rangle \rightarrow\langle K, S\rangle$ in $\mathscr{S}(G)$ (where $H^{i} \supset K^{i}$ and $H \supset K$ ). To prove that $\left\langle H^{i},_{H^{i}} U^{S^{i}}\right\rangle \rightarrow\left\langle H_{H_{H}} U^{S}\right\rangle$, it suffices by Proposition 1.2 to show that the set $\mathscr{R}$ of all $\left\langle H^{i},{ }_{H^{i}} U^{S^{i}}\right\rangle$ weakly contains $\left\langle H,{ }_{H} U^{S}\right\rangle$. Since $S$ is a direct sum of cyclic representations, it is sufficient to make the proof assuming $S$ to be cyclic. Let $\phi$ be the function of positive type on $K$ associated with a cyclic vector for $S$. By Theorem 3.1' we may replace $\left\{\left\langle K^{i}, S^{i}\right\rangle\right\}$ by a subnet, and choose for each $i$ a sum $\phi_{i}$ of functions of positive type on $K^{i}$ associated with $S^{i}$, so that $\phi_{i} \rightarrow \phi$ in $\mathscr{E}_{s}(G)$.

By Theorem 4.1 the measure $\rho$, defined on $K$ by $d \rho x=\left(\Delta_{H}(x) / \Delta_{K}(x)\right)^{1 / 2} \phi(x) d \mu_{K} x$, is of positive type on $H$ when considered as a measure on $H$, and $T^{\rho} \cong{ }_{H} U^{S}$. Similarly $\rho^{i}$, defined on $K^{i}$ by $d \rho^{i} x=\left(\Delta_{H^{i}}(x) / \Delta_{K^{i}}(x)\right)^{1 / 2} \phi_{i}(x) d \mu_{K^{i}} x$, is of positive type on $H^{i}$, and $T^{\rho^{i}}$ is a subrepresentation of a direct sum of finitely many copies of ${ }_{H^{i}} U^{S^{t}}$.

Fix an element $f$ in $C_{0}(H)$, and let $\xi=\Phi_{H, \rho}(f) \in H\left(T^{\rho}\right)$. Choose an element $F$ of $C_{0}(G)$ whose restriction to $H$ is $f$; set $f_{i}=F \mid H^{t}$ for each $i$; and let $\xi_{i}=\Phi_{H^{i}, \rho^{i}}\left(f_{i}\right) \in H\left(T^{\rho^{i}}\right)$. For each $h$ in $C_{0}(Y)$ we verify

$$
\left(T_{h}^{\rho^{i}} \xi_{i}, \xi_{i}\right)=\int_{H^{i}}\left(F^{*} * h * F\right)\left(H^{i}, x\right) d \rho^{i} x,
$$


where $F^{*} * h * F$ is evaluated in the ${ }^{*}$-algebra $C_{0}(Y)$ (regarding $F$ as an element of $\left.C_{0}(Y): F(L, x)=F(x)\right)$. Substituting for $\rho^{i}$ we get

$$
\left(T_{h}^{\rho^{i}} \xi_{l}, \xi_{i}\right)=\int_{K^{i}}\left(F^{*} * h * F\right)\left(H^{i}, x\right)\left(\Delta_{H^{i}}(x) / \Delta_{K^{i}}(x)\right)^{1 / 2} \phi_{i}(x) d \mu_{K^{i}} x .
$$

Let $E$ be an element of $C_{0}(G)$ such that $E(x)=\left(F^{*} * h * F\right)(H, x)$ whenever $x \in H$. Then for each $\varepsilon>0$ we have $i$-eventually

$$
\left|\left(F^{*} * h * F\right)\left(H^{i}, x\right)-E(x)\right|<\varepsilon \text { for all } x \text { in } H^{i} .
$$

Using Proposition 3.1, and recalling that $\Delta_{L}(x)$ is continuous in $L$ and $x$, we find

$$
\begin{aligned}
\lim _{i} \int_{K^{i}} E(x)\left(\Delta_{H^{i}}(x) / \Delta_{K^{i}}(x)\right)^{1 / 2} \phi_{i}(x) d \mu_{K^{i}} x \\
=\int_{K}\left(F^{*} * h * F\right)(H, x)\left(\Delta_{H}(x) / \Delta_{K}(x)\right)^{1 / 2} \phi(x) d \mu_{K^{\prime}} x \\
=\int_{K}\left(F^{*} * h * F\right)(H, x) d \rho x=\left(T_{h}^{\rho \xi}, \xi\right) .
\end{aligned}
$$

Combining (1), (2), and (3), we obtain $\lim _{i}\left(T_{h}^{\rho^{\prime}} \xi_{i}, \xi_{i}\right)=\left(T_{h}^{\rho} \xi, \xi\right)$.

From this and the arbitrariness of $h$ it follows that the positive functional on $C_{s}^{*}(G)$ associated with the vector $\xi$ in $H\left(T^{\rho}\right)$ can be weakly*-approximated by positive functionals associated with the $\left\langle H^{i}, T^{\rho^{i}}\right\rangle$, that is, finite sums of positive functionals associated with $\mathscr{R}$. But these $\xi$ are dense in $H\left(T^{\rho}\right)$. So $\left\langle H, T^{\rho}\right\rangle$ is weakly contained in $\mathscr{R}$, which was all we had to prove.

As a corollary of this we obtain:

TheOREM 4.3. Let $Q$ be a subset of $\mathscr{S}(G)$ and $\langle K, T\rangle$ an element of $\mathscr{S}(G)$ weakly contained in $Q$. Let $L$ be a closed subgroup of $G$ such that $L \supset K$ and $L \supset K^{\prime}$ for all $\left\langle K^{\prime}, T^{\prime}\right\rangle$ in $Q$. Then ${ }_{L} U^{T}$ is weakly contained in the family $o$ all ${ }_{L} U^{T^{\prime}}$, where $\left\langle K^{\prime}, T^{\prime}\right\rangle$ ranges over $Q$.

The proof is similar to that of Theorem 3.3.

Theorems 4.1 and 4.2 of [5] are of course very special cases of Theorems 4.2 and 4.3 above.

5. Topological Frobenius properties. Throughout this section the locally compact group $G$ is assumed to satisfy the second axiom of countability. Otherwise the notation remains as before.

We shall contemplate the following topological "Frobenius Property" (FP) which $G$ may possess :

\footnotetext{
( 1$)$ In the equation that follows we should strictly write $W^{H^{t}, T^{\rho i}}(h)\left(\xi_{i}\right)$ instead of $T^{\rho t}\left(\xi_{i}\right)$; but the meaning is clear.
} 
(FP) $\left({ }^{22}\right)$. Whenever $K \in \mathscr{K}(G), T \in \mathcal{K}$, and $S \in \hat{G},{ }_{G} U^{T}$ weakly contains $S$ if and only if $S \mid K$ weakly contains $T$.

Not only this property, but very much weaker properties fail to hold if $G$ is arbitrary. However, we begin by observing:

THEOREM 5.1. (FP) holds if $G$ is compact or Abelian.

Proof. If $G$ is compact, weak containment is the same as ordinary containment; so (FP) follows from the classical Frobenius theorem for compact groups. If $G$ is Abelian we apply Corollary 2, p. 261 of [5] and Theorem 3.2, p. 255 of [5].

In view of the recent work of Kirillov [13], it appears likely that (FP) holds for connected nilpotent Lie groups also.

(FP) implies the following two weaker properties:

(WF1). For each closed subgroup $K$ of $G$ and each $S$ in $\hat{G},{ }_{G} U{ }^{S \mid K}$ weakly contains $S$.

(WF2). For each closed subgroup $K$ of $G$ and each $T$ in $\hat{K},{ }_{G} U^{T} \mid K$ weakly contains $T$.

Let us derive (WF1) assuming (FP). Let $S$ and $K$ be as in (WF1); and choose any $T$ in $\hat{K}$ such that $S \mid K$ weakly contains $T$. By Theorem 4.3, $U^{S \mid K}$ weakly contains $U^{T}$; and by (FP) $U^{T}$ weakly contains $S$. So $U^{S \mid K}$ weakly contains $S$.

Similarly we derive (WF2) from (FP) by appealing to Theorem 3.3.

(WF2) implies the still weaker property:

(WF3). If $K$ is a closed subgroup of $G$, every $T$ in $\hat{K}$ is weakly contained in $S \mid K$ for some unitary representation $S$ of $G$.

It will be seen in $\S 6$ that even (WF3) fails for general $G$.

Taking $K=\{e\}$ in (WF1) we see that (WF1) can hold only if the regular representation of $G$ weakly contains all $S$ in $\hat{G}$. The latter condition fails for the connected semisimple noncompact Lie groups (see [21]), and also for the (discrete) free group on two generators (see [23]). So (WF1) fails in general. We do not know whether (WF1) is equivalent to the condition that the regular representation weakly contains all of $\hat{G}$.

Proposition 5.1. (WF1) holds if and only if, for each $K$ in $\mathscr{K}(G),{ }_{G} U^{J}$ weakly contains $I$, where $J$ and $I$ are the identity representations of $K$ and $G$, respectively.

Proof. The condition is obviously necessary. Assume that it holds, and that $K \in \mathscr{K}(G)$ and $S \in \hat{G}$. Let $J$ and $I$ be as above. By [5, Corollary 1, p. 260], $U^{S \mid K} \cong U^{J} \otimes S$. By assumption $U^{J}$ weakly contains $I$. So (see [8]) $U^{J} \otimes S$ weakly contains $I \otimes S=S$.

Discrete groups satisfy (WF2), and hence (WF3). In fact we easily verify:

(22) See [22] for the classical Frobenius Theorem for compact groups. Our "Frobenius Property" cannot contain any reference to the multiplicities of the representations, since there seems to be no reasonable definition of " $S$ weakly containing $T \boldsymbol{n}$ times." 
Proposition 5.2. Let $K$ be a subgroup of $G$ which is both open and closed in $G$. Then, for each unitary representation $S$ of $K, S$ is a direct summand of ${ }_{G} U^{S} \mid K$.

However, as we have already pointed out (see [23]), discrete groups need not satisfy (WF1).

It follows from [5, Theorem 4.5], that (WF2) holds if only normal subgrou ps are considered.

Proposition 5.3. If $K$ is a closed normal subgroup of $G$, then ${ }_{G} U^{T} \mid K$ weakly contains $T$ for all $T$ in $\hat{K}$.

Next we deduce a topological version of Mackey's Restriction Theorem [14, Theorem 12.1].

THEOREM 5.2. Let $G$ be a separable locally compact group, and let $K$ and $L$ be two closed subgroups of $G$ such that (a) $K$ and L are regularly related in the sense of Mackey [14, p. 127], and (b) $K \cap L$ has property (WF1). Then for each unitary representation $T$ of $K$,

$$
{ }_{G} U^{T} \mid L \text { weakly contains }{ }_{L} U^{T \mid K \cap L} .
$$

Proof. Let $\mathscr{D}$ be the family of all $K, L$ double cosets $K x L(x \in G)$, and $v$ any admissible measure in $\mathscr{D}[14$, p. 127]. Then, by $[14$, Theorem 12.1],

$$
U^{T} \mid L \cong \int_{\mathscr{D}} D V d v D
$$

where, for each $D$ in $\mathscr{D},{ }_{x} V={ }_{D} V$ is the unitary representation of $L$ induced from the representation $u \rightarrow T_{x u x^{-1}}$ of $x^{-1} K x \cap L$ (here $x$ is any element of $D$ ).

Let $Q={ }_{L} U^{T \mid K \cap L}$. I claim that, if $x_{n} \rightarrow e$ in $G$, then

$$
{ }_{x_{n}} V \rightarrow Q
$$

in the inner hull-kernel topology of representations of $L$.

Indeed, by Proposition 1.2, it is enough to show that the set of all ${ }_{x_{n}} V$ weakly contains $Q$. Now let $L_{n}=x_{n}^{-1} K x_{n} \cap L$, and let $S^{n}$ be the representation $u \rightarrow T_{x_{n} u x_{n}-1}$ of $L_{n}$. Since $\mathscr{K}(L)$ is compact, we may pass to a subsequence and assume that $L_{n} \rightarrow L^{\prime} \subset L$ in the compact-open topology. Since $x_{n} \rightarrow e, L^{\prime} \subset L \cap K$. Let $S=T \mid L^{\prime}$. Combining Lemma 2.7 (applied to the group $M$ of inner automorphisms of $G$ ) with Theorem 3.2 , we easily see that $\left\langle L_{n}, S^{n}\right\rangle \rightarrow\left\langle L^{\prime}, S\right\rangle$ in $\mathscr{S}(G)$. Hence by Theorem 4.2

$$
\left\langle L,{ }_{x_{n}} V\right\rangle=\left\langle L,{ }_{L} U^{S^{n}}\right\rangle \rightarrow\left\langle L,{ }_{L} U^{S}\right\rangle
$$

in $\mathscr{S}(G)$. Since $L^{\prime} \subset L \cap K$ and $L \cap K$ satisfies (WF1), ${ }_{(L \cap K)} U^{S}$ weakly contains $T \mid(L \cap K)$. So by Theorem $4.3,{ }_{L} U^{S}$ weakly contains ${ }_{L} U^{T \mid L \cap K}=Q$. This combined with (3) (and Proposition 1.3) gives (2). 
Now let $\mathscr{Z}$ be an arbitrary inner hull-kernel neighborhood of $Q$ in $\mathscr{T}(L)$. In view of (2) there is an open neighborhood $W$ of $e$ in $G$ such that, if $x \in W$, ${ }_{x} V \in \mathscr{Z}$. Thus the inverse image of $\mathscr{Z}$ under the mapping $D \rightarrow_{D} V$ contains the set of all $K, L$ double cosets intersecting $W$, and this set is of positive $v$ measure. Hence by (1) and [5, Theorem 3.1], $U^{T} \mid L$ weakly contains $Q$. This completes the proof.

If in the preceding theorem we replace the unit element by any fixed $x$ in $G$, and assume that $x^{-1} K x \cap L$ has Property (WF1), the same proof leads to the conclusion that ${ }_{G} U^{T} \mid L$ weakly contains ${ }_{L} U^{T^{x} \mid\left(x^{-1} K x \cap L\right)}$ (where $T_{u}^{x}=T_{x u x^{-1}}$ ). In view of [5, Theorem 3.1], we thus obtain:

THEOREM 5.3. Suppose that $K$ and L are two regularly related closed subgroups of $G$, and that $x^{-1} K x \cap L$ has Property (WF1) for all $x$ in $G$. Then ${ }_{G} U^{T} \mid$ Lis weakly equivalent to the set of all ${ }_{L} U^{T^{x} \mid\left(x^{-1} K x \cap L\right)}$, where $x$ runs over $G$.

Just as [14, Theorem 12.1] led immediately to [14, Theorem 12.2] on Kronecker products of representations, so from our Theorems 5.2 and 5.3 we deduce the following theorems on Kronecker products:

THEOREM 5.4. Let $K$ and $L$ be two regularly related closed subgroups of $G$ such that $K \cap L$ has Property (WF1). If $S$ and $T$ are unitary representations of $K$ and $L$, respectively, then ${ }_{G} U^{S} \otimes{ }_{G} U^{T}$ weakly contains ${ }_{G} U^{(S|(K \cap L) \otimes T|(K \cap L))}$.

THEOREM 5.5. Let $K$ and $L$ be two regularly related closed subgroups of $G$, such that $x^{-1} K x \cap L$ has Property (WF1) for all $x$ in $G$. Then, if $S$ and $T$ are unitary representations of $K$ and $L$, respectively, ${ }_{G} U^{S} \otimes{ }_{G} U^{T}$ is weakly equivalent to the set of all ${ }_{G} U^{\left(S^{x}\left|\left(x^{-1} K x \cap L\right) \otimes T\right|\left(x^{-1} K x \cap L\right)\right)}$, where $x$ runs over $G$.

Theorem 5.2 has a number of interesting special cases:

COROLlaRY 1. If $K$ is a subgroup of $G$ which is regularly related to itself and satisfies (WF1), then ${ }_{G} U^{T} \mid K$ weakly contains $T$ for all unitary representations $T$ of $K$.

If $K$ and $L$ are closed subgroups of $G$ one of which is compact, then $K$ and $L$ are necessarily regularly related. Also, a compact group satisfies (WF1) by Theorem 5.1. So we have:

COROLlary 2. If $K$ is a compact subgroup of $G, L$ is a closed subgroup of $G$, and $T$ and $S$ are unitary representations of $K$ and $L$, respectively, then ${ }_{G} U^{T} \mid L$ weakly contains ${ }_{L} U^{T \mid K \cap L}$, and ${ }_{G} U^{S} \mid K$ weakly contains ${ }_{R} U^{S \mid K \cap L}$.

In particular, if $K=L$, we get:

COROLlaRY 3. If $K$ is a compact subgroup of $G$, and $T$ is a unitary representation of $K$, then ${ }_{G} U^{T} \mid K$ weakly contains $T$. 
COROLlary 4. If $K$ is a compact subgroup of $G, L$ is a closed subgroup of $G, K \cap L=\{e\}$, and $T$ is a unitary representation of $K$, then ${ }_{G} U^{T} \mid L$ weakly contains the regular representation of $L$.

Putting $K=\{e\}$ in Corollary 4, we find:

COROLlary 5. If $L$ is any closed subgroup of $G$, the regular representation of $G$, when restricted to $L$, weakly contains the regular representation of $L$.

From Corollary 5 and Theorem 2.2 we obtain :

COROLlaRy 6. The subgroup representation theory of $G$ is continuous at a closed subgroup $K$ of $G$ provided that the regular representation of $K$ weakly contains the identity representation of $K$ (and hence all irreducible representations of $K$; see $[12 ; 21])$.

6. A counter-example to (WF3). This section contains a counter-example to (WF3). That is, we exhibit a group $G$, a closed subgroup $K$, and an irreducible unitary representation $T$ of $K$ which is not weakly contained in $S \mid K$ for any unitary representation $S$ of $G$. For $G$ we take the $3 \times 3$ complex unimodular group $\operatorname{SL}(3, C)$, and for $K$ the group $\operatorname{SL}(2, C)$, which we identify with the subgroup of $\operatorname{SL}(3, C)$ consisting of those $a$ for which $a_{13}=a_{23}=a_{31}=a_{32}=0$, $a_{33}=1$. $T$ will be any member of the supplementary series of irreducible representations of $\operatorname{SL}(2, C)$.

Let $M$ be the subgroup of $\operatorname{SL}(3, C)$ consisting of those $a$ for which $a_{13}=a_{23}=0, a_{33}=1 . M$ is a semidirect product of the closed normal Abelian subgroup $N$ consisting of all

$$
c_{s}=\left(\begin{array}{lll}
1 & 0 & 0 \\
0 & 1 & 0 \\
r & s & 1
\end{array}\right] \quad(r, s \text { complex })
$$

and the closed subgroup $\operatorname{SL}(2, C)$. The character group $\hat{N}$ of $N$ has just two orbits under the action of $M$, the orbit consisting of the identity character only and the orbit containing everything else. One element of the second orbit is $\chi$, where

$$
\chi\left(c_{r . s}\right)=e^{i \operatorname{Re}(r)} .
$$

The "stationary subgroup" $Z$ of $\chi$ in $M$ consists of all

$$
a=\left(\begin{array}{lll}
1 & z & 0 \\
0 & 1 & 0 \\
r & s & 1
\end{array}\right) \quad(r, s, z \text { complex })
$$

and the irreducible representations of $\mathrm{Z}$ which reduce on $N$ to multiples of $\chi$ are exactly the characters $\psi_{w}$ ( $w$ complex $)$, where 


$$
\psi_{w}(a)=e^{i \operatorname{Re}(r+z \bar{w}}
$$

( $a$ being as in (1)). So, by Mackey's theory of induced representations [14, Theorem 14.1], the irreducible representations of $M$ fall into two classes:

(i) the $V^{w}={ }_{M} U^{\psi w}$ ( $w$ complex);

(ii) those lifted from irreducible representations of $\operatorname{SL}(2, C)$.

Now the irreducible representations of $\operatorname{SL}(2, C)$ fall into three disjoint classes the principal series, the supplementary series, and the one-dimensional identity representation (see [9]). It was shown in [4] that the principal series is closed in $\operatorname{SL}(2, C)^{\wedge}$, while the supplementary series is open.

LEMMA 6.1. The set of all $V^{w} \mid \operatorname{SL}(2, C)$ ( $w$ complex) is weakly contained in the principal series of irreducible representations of $\operatorname{SL}(2, C)$.

Proof. By Mackey's Restriction Theorem [14, Theorem 12.1], $V^{w} \mid \operatorname{SL}(2, C)$ is equivalent to the representation of $\operatorname{SL}(2, C)$ induced from a character of $Z \cap S L(2, C)=Z^{\prime}$. Since $Z^{\prime}$ is Abelian, its characters are weakly contained in its regular representation. Hence by our Theorem 4.2 and [14, Theorem 4.2], each $V^{w} \mid \operatorname{SL}(2, C)$ is weakly contained in the regular representation of $\operatorname{SL}(2, C)$. But the latter, by [4, Theorem 3.2], is weakly equivalent to the principal series. Hence the conclusion of the lemma holds.

THEOREM 6.1. If $S$ is any unitary representation of $\operatorname{SL}(3, C)$ and $T$ is any member of the supplementary series of irreducible representations of $\operatorname{SL}(2, C)$ then $S \mid \operatorname{SL}(2, C)$ does not weakly contain $T$.

Proof. Since $S$ is weakly contained in $\operatorname{SL}(3, C)^{\wedge}$, it is enough by Theorem 3.3 to prove that $T$ is not weakly contained in the set of all $S \mid \operatorname{SL}(2, C)$, where $S$ runs over $\operatorname{SL}(3, C) \wedge$.

Now $\operatorname{SL}(3, C)^{\wedge}$ is well known $[1 ; 20]$; it consists of the irreducible representations constructed in [9]. Thus, taking any irreducible unitary representation $S$ of $\operatorname{SL}(3, C)$ other than the trivial one, one verifies directly that $H(S)$ contains no nonzero vector invariant under each $a$ in $N$. On the other hand, since $M$ is of Type $I$ and has smooth dual (see $[14 ; 10]), S \mid M$ can be decomposed as a direct integral over $\hat{M}$ (see [16]); and the absence of $N$-invariant vectors shows that in this direct integral the elements of $\hat{M}$ lifted from elements of $\operatorname{SL}(2, C)$ ^ occur with measure 0 . Thus, by [5, Theorem 3.1], $S \mid M$ is weakly contained in $\left\{V^{w} \mid w\right.$ complex $\}$. Combining this fact with Lemma 6.1 and Theorem 3.3, we deduce that $S \mid \operatorname{SL}(2, C)$ is weakly contained in the principal series of $\operatorname{SL}(2, C) \wedge$. Thus every $S$ in $\operatorname{SL}(3, C)^{\wedge}$, when restricted to $\operatorname{SL}(2, C)$, is weakly contained in the complement of the supplementary series in $\operatorname{SL}(2, C) \wedge$. Since this complement is closed [4], we obtain the theorem. 


\section{BIBLIOGRA PHY}

1. F. A. Berezin, Laplace operators on semisimple Lie groups, Trudy Moskov. Mat. Obšč. 6 (1957), 371-463.

2. R. J. Blattner, On induced representations, Amer. J. Math. 83 (1961), 79-98.

3. __ Positive definite measures, Proc. Amer. Math. Soc. 14 (1963), 423-428.

4. J. M. G. Fell, The dual spaces of $C^{*}$-algebras, Trans. Amer. Math. Soc. 94 (1960), 365-403.

5. - Weak containment and induced representations of groups, Canad. J. Math. 14 (1962), 237-268.

6. - The structure of algebras of operator fields, Acta Math. 106 (1961), 233-280.

7. — A Hausdorff topology for the closed subsets of a locally compact non Hausdorff space, Proc. Amer. Math. Soc. 13 (1962), 472-476.

8. - Weak containment and Kronecker products of group representations, Pacific J. Math. 13 (1963), 503-510.

9. I. M. Gelfand and M. A. Naimark, Unitary representations of the classical groups, Trudy Mat. Inst. Steklov. 1950.

10. J. G. Glimm, Type I $C^{*}$-algebras, Ann. of Math. (2) 73 (1961), 572-612.

11. —_, Families of induced representations, Pacific J. Math. 12 (1962), 885-911.

12. R. Godement, Les fonctions de type positif et la théorie des groupes, Trans. Amer. Math. Soc. 63 (1948), 1-84.

13. A. A. Kirillov, Unitary representations of nilpotent Lie groups, Uspehi. Mat. Nauk 17 (1962), 57-110.

14. G. W. Mackey, Induced representations of locally compact groups. I, Ann. of Math. (2) 55 (1952), 101-139.

15. - Induced representations of locally compact groups. II. The Frobenius reciprocity theorem, Ann. of Math. (2) 58 (1953), 193-221.

16. _ Borel structure in groups and their duals, Trans. Amer. Math. Soc. 85 (1957), 134-165.

17. - Unitary representations of group extensions. I, Acta Math. 99 (1958), 265-311.

18. F. I. Mautner, A generalization of the Frobenius reciprocity theorem, Proc. Nat. Acad. Sci. U.S.A. 37 (1951), 431-435.

19. M. A. Naimark, Normed rings, GITTL, Moscow, 1956.

20. - Description of all irreducible unitary representations of the classical groups, Dokl. Akad. Nauk SSSR 84 (1952), 883-886.

21. O. Takenouchi, Sur une classe de fonctions continues de type positif sur un groupe localement compact, Math. J. Okayama Univ. 4 (1955), 143-173.

22. A. Weil, L'intégration dans les groupes topologiques et ses applications, Hermann, Paris, 1940.

23. H. Yoshizawa, Some remarks on unitary representations of the free group, Osaka Math. J. 3 (1951), 55-82.

\section{UNIVERSITY OF WASHINGTON,} Seattle, Washington 